\title{
Impact of Health Insurance on Stage at Cancer Diagnosis
} Among Adolescents and Young Adults

\author{
Theresa H. M. Keegan, Helen M. Parsons, Yi Chen, Frances B. Maguire, Cyllene R. Morris, \\ Arti Parikh-Patel, Kenneth W. Kizer, Ted Wun
}

See the Notes section for the full list of authors' affiliations.

Correspondence to: Theresa H. M. Keegan, PhD, Division of Hematology and Oncology, UC Davis Comprehensive Cancer Center, 4501 X Street, Suite 3016, Sacramento, CA 95817 (e-mail: tkeegan@ucdavis.edu).

\begin{abstract}
Background: Uninsured adolescents and young adults (AYAs) and those with publicly funded health insurance are more likely to be diagnosed with cancer at later stages. However, prior population-based studies have not distinguished between AYAs who were continuously uninsured from those who gained Medicaid coverage at the time of cancer diagnosis.

Methods: AYA patients (ages 15-39 years) with nine common cancers diagnosed from 2005 to 2014 were identified using California Cancer Registry data. This cohort was linked to California Medicaid enrollment files to determine continuous enrollment, discontinuous enrollment, or enrollment at diagnosis, with other types of insurance determined from registry data. Multivariable logistic regression was used to evaluate factors associated with later stages at diagnosis.

Results: The majority of 52774 AYA cancer patients had private or military insurance (67.6\%), followed by continuous Medicaid (12.4\%), Medicaid at diagnosis (8.5\%), discontinuous Medicaid (3.9\%), other public insurance (1.6\%), no insurance (2.9\%), or unknown insurance (3.1\%). Of the 13069 with Medicaid insurance, $50.1 \%$ were continuously enrolled. Compared to those who were privately insured, AYAs who enrolled in Medicaid at diagnosis were 2.2-2.5 times more likely to be diagnosed with later stage disease, whereas AYAs discontinuously enrolled were 1.7-1.9 times and AYAs continuously enrolled were 1.4-1.5 times more likely to be diagnosed with later stage disease. Males, those residing in lower socioeconomic neighborhoods, and AYAs of Hispanic or black race and ethnicity (vs non-Hispanic white) were more likely to be diagnosed at a later stage, independent of insurance.

Conclusions: Our findings suggest that access to continuous medical insurance is important for decreasing the likelihood of late stage cancer diagnosis.
\end{abstract}

Cancer incidence in adolescents and young adults (AYAs: ages 15-39 years) is increasing, and it is the leading cause of nonaccidental deaths in this age group $(1,2)$. Although survival has substantially improved for pediatric and older cancer patients, less improvement has occurred among AYAs $(3,4)$. One factor contributing to less survival improvements is more limited access to health care, as AYAs have historically been the most highly uninsured group in the United States $(3,5)$.

Nationally representative studies of AYA cancer patients have found that lacking insurance or having publicly funded health insurance at diagnosis or initial treatment are associated with being diagnosed at a later stage, being undertreated, and experiencing worse survival (6-8). However, these studies were unable to distinguish between those who were uninsured from those who became publicly insured through Medicaid at the time of cancer diagnosis. In many states, including California, Medicaid coverage is extended to eligible uninsured patients after a cancer diagnosis. Because those who were uninsured prior to diagnosis may not have had cancer screening and other nonemergent care (9), it is important to differentiate patients continuously enrolled in Medicaid from those who enroll at the time of cancer diagnosis. Indeed, among older adults with 
selected cancers, Medicaid enrollment at diagnosis (vs being enrolled at least sixmonths prior to diagnosis) was associated with being diagnosed at more advanced stages, with fewer definitive operations, and with higher one-year mortality (9).

To date, no previous study has focused on how continuous vs newly gained Medicaid enrollment influences stage at diagnosis compared with privately insured AYAs to estimate the potential gap in access to care. Therefore, we linked Medi-Cal (ie, California's Medicaid program) enrollment files to California Cancer Registry (CCR) data for AYAs diagnosed with the most common cancers during 2005-2014 to examine the impact of prior health insurance on stage at diagnosis, after adjustment for other demographic and clinical variables known to be associated with stage at diagnosis.

\section{Materials and Methods}

\section{Study Population}

Patients eligible for the study were all persons who resided in California when diagnosed at age 15-39years with a first primary, invasive, histologically confirmed cancer from March 1, 2005, through December 1, 2014 (to coincide with Medi-Cal enrollment data available from October 1, 2004, through December $1,2014)$ and reported to the CCR. As done previously (10), we focused on nine common cancers in the US AYA population with stage defined by the American Joint Commission on Cancer (AJCC), sixth edition: female breast carcinoma, thyroid carcinoma, melanoma, testicular cancer, Hodgkin lymphoma (HL), non-Hodgkin lymphoma (NHL), colorectal cancer, cervical cancer, and ovarian cancer. The Surveillance, Epidemiology, and End Results (SEER) AYA recode, based on the AYA classification suggested by Barr et al. (11) and updated based on histology changes in the World Health Organization (WHO) Classification of Tumours of Hematopoietic and Lymphoid Tissues, was primarily used to determine histologic types of cancer. For those primary sites not individually designated in the AYA recode listing (testicular, ovarian, cervical, colorectal cancers), we utilized the SEER site recode (ICD-O-3/WHO 2008) (12).

From the CCR, which operates under a state cancer reporting law and comprises three National Cancer Institute (NCI) SEER registries, we obtained information routinely recorded in the medical record at diagnosis for each patient on age, sex, race and ethnicity, stage at diagnosis, marital status, health insurance, hospital providing initial care (NCI-designated cancer center or not), and census-block group of residence. A Deyo and Romano-adapted Charlson comorbidity index was obtained from the CCR (13). For comparison, stage at diagnosis was classified as both AJCC stage I vs II-IV and stage I-II vs III-IV. For sensitivity analyses, we classified AYAs with unknown stage into AJCC stage categories with similar five-year survival for each cancer type (14) and considered AJCC stage I vs stage II, III, and IV separately.

We used a multicomponent index of neighborhood socioeconomic status (SES) based on patients' residential census-block group at diagnosis. The index is derived from data from the 2000 US Census and the 2006-2010 American Community Survey on education, occupation, unemployment, household income, poverty, rent, and house values (15) and is grouped into quintiles based on the distribution of SES across all censusblock groups in California. Rural/urban designations are based on Medical Service Study Area from the 2000 and 2010 US Census.
Using a deterministic strategy, the California Department of Health Care Services linked CCR data to monthly Medi-Cal enrollment files, with linkage methods reported previously (9). Health insurance, defined as the primary source of payment at diagnosis or initial treatment, is routinely abstracted from the CCR. For this study, we utilized primary health insurance information from the earliest record in the CCR. Focusing on enrollment six months prior to and six months after diagnosis, linkage to the Medi-Cal enrollment files allowed for the mutually exclusive classification of 1) continuous enrollees, defined as enrolled five or six months prior to diagnosis; 2) Medicaid at diagnosis, defined as coverage beginning in the month prior to or within two months after diagnosis to account for reactive enrollment; and 3) discontinuous Medicaid coverage, defined as enrollment that does not meet the definitions for continuous enrollees or Medicaid at diagnosis or Medicaid insurance recorded in the CCR but without a match in the Medi-Cal enrollment files ( $1 \%$ of patients in this category) (Supplementary Figure 1, available online). From the CCR, we additionally classified AYAs into other public insurance (Medicare, Indian/Public Health Service, county-funded not otherwise specified), privately insured (health maintenance organizations, preferred provider organizations, managed care not otherwise specified, and Department of Defense [Tricare, military treatment facilities]), uninsured, and other and unknown.

The final study population included 52774 AYA cancer patients after exclusion of 373 patients reported by Veterans Affairs medical centers (Supplementary Figure 1, available online). These analyses were performed under a research protocol approved by the California's Health and Human Services Agency Committee for the Protection of Human Subjects.

\section{Statistical Analyses}

The outcome of interest was later stage at diagnosis, defined as AJCC stage II-IV and III-IV. To evaluate associations with later stage at diagnosis, we used multivariable logistic regression to estimate odds ratios (ORs) and associated 95\% confidence intervals (CIs). Models were built for all patients and those with Medicaid insurance only and included variables with a priori reasons for inclusion (eg, age, race and ethnicity, gender, year of diagnosis, marital status, facility type, neighborhood SES and urban/rural residence, and health insurance type). Multicollinearity in our models was assessed by examining variation inflation factors (VIF). All models met our criteria of nonmulticollinearity with VIF less than 10 . Effect modification was assessed between health insurance, cancer type, and year of diagnosis by including interaction terms in the multivariable models. As effect modification was evident by cancer type, but not year of diagnosis, separate logistic regression models were conducted by cancer type. The main analyses excluded patients with missing stage at diagnosis $(n=2739)$. Regression analyses were conducted using SAS version 9.4 software, SAS Institute Inc., Cary, NC. All statistical tests were two-sided, and 95\% confidence intervals that did not cross 1.00 were considered statistically significant.

\section{Results}

The majority of the 52774 AYA cancer patients had private or military insurance (67.6\%) followed by continuous Medicaid (12.4\%), Medicaid at diagnosis (8.5\%), discontinuous Medicaid (3.9\%), no insurance $(2.9 \%)$ or unknown insurance $(3.1 \%)$, and 
other public insurance (1.6\%). Of the 13069 with Medicaid insurance, $50.1 \%$ were continuously enrolled. Sociodemographic and clinical characteristics of AYA patients varied by type of health insurance, with a higher proportion of non-Hispanic whites and Asian/Pacific Islanders with private or military insurance and higher proportions of blacks and Hispanics with Medicaid insurance (Table 1). AYAs with private or military health insurance were more likely to reside in the highest two categories of neighborhood SES and be diagnosed with stage I-II disease than AYAs with other types of insurance. AYAs who remained uninsured were more likely to be male, diagnosed with cancer from 2005 to 2009, and diagnosed with stage I-II disease than those who obtained Medicaid insurance at diagnosis.

In multivariable models of all cancers combined, health insurance type was statistically significantly associated with later stage at cancer diagnosis (Table 2). Compared to AYAs with private health insurance, AYAs who gained Medicaid coverage at diagnosis were 2.2-2.5 times more likely to be diagnosed at a later stage (stage II-IV vs I: OR $=2.46,95 \% \mathrm{CI}=2.26$ to 2.69 ; III-IV vs I-II: $\mathrm{OR}=2.16,95 \% \mathrm{CI}=2.00$ to 2.33 ), whereas AYAs with discontinuous Medicaid were 1.7-1.9 times more likely to be diagnosed at a later stage (stage II-IV vs I: OR $=1.93,95 \% \mathrm{CI}=1.70$ to 2.18; III-IV vs I-II: $\mathrm{OR}=1.74,95 \% \mathrm{CI}=1.56$ to 1.95 ). AYAs with continuous Medicaid insurance were 1.4-1.5 times more likely and AYAs with no insurance were 1.2 times more likely to be diagnosed at a later stage. Associations between health insurance and stage at diagnosis were similar in models that imputed unknown stage at diagnosis (Supplementary Table 1, available online), and associations with health insurance were stronger with each level of AJCC stage (Supplementary Table 2, available online).

Blacks and Hispanics (vs non-Hispanic whites) had higher odds of later stage at diagnosis (Table 2). In contrast, Asian/ Pacific Islanders and American Indian/Alaskan were not more likely than non-Hispanic whites to be diagnosed with later stage disease. AYAs with comorbidities or residing in the lowest three categories of neighborhood SES were more likely to be diagnosed with later stage disease. On the other hand, females and those diagnosed in 2010-2014 were less likely to be diagnosed with later stage disease.

In analyses limited to AYAs with Medicaid insurance (Supplementary Table 3, available online), those who gained Medicaid coverage at diagnosis were approximately 1.5-1.6 times more likely to be diagnosed at a later stage than those continuously enrolled in Medicaid (stage II-IV vs I: OR $=1.63$, $95 \% \mathrm{CI}=1.47$ to 1.81 ; III-IV vs I-II: $\mathrm{OR}=1.49,95 \% \mathrm{CI}=1.36$ to 1.64). AYAs with discontinuous (vs continuous) Medicaid also were more likely to be diagnosed at a later stage (stage II-IV vs I: $\mathrm{OR}=1.28,95 \% \mathrm{CI}=1.12$ to 1.46 ; III-IV vs $\mathrm{I}-\mathrm{II}$ : $\mathrm{OR}=1.20,95 \%$ $\mathrm{CI}=1.06$ to 1.36). Associations between other factors and stage at diagnosis in AYAs with Medicaid insurance were similar to the model with AYAs with all types of health insurance, with the exception that associations among Hispanics and by neighborhood SES were less pronounced.

In multivariable models by cancer type, Medicaid at diagnosis was associated with later stage at diagnosis across all nine cancers considered (Table 3). Discontinuous Medicaid was associated with later stage at diagnosis for all cancer sites, except for thyroid cancer where there was a borderline association. In addition, compared to AYAs who were privately insured, AYAs without insurance or with continuous Medicaid also had a higher odds of later stage at diagnosis that varied somewhat by cancer site. In particular, being uninsured was associated with later stage at diagnosis for AYAs diagnosed with $\mathrm{HL}$ and cervical cancer, and continuous Medicaid enrollment was associated with later stage at diagnosis for AYAs diagnosed with breast cancer, melanoma, testicular cancer, NHL, cervical cancer, and ovarian cancer. Associations were similar in models that imputed unknown stage at diagnosis (Supplementary Table 4, available online).

\section{Discussion}

In this population-based study of more than 52000 AYAs diagnosed with nine common invasive cancers, those enrolled in Medicaid were more likely to be diagnosed at a later stage than AYAs with private health insurance. The duration and continuity of Medicaid enrollment influenced these associations, with AYAs who enrolled at diagnosis more than 2.0 times, AYAs intermittently enrolled 1.7 to 1.9 times, and AYAs continuously enrolled 1.4-1.5 times more likely to have later stage disease than those with private insurance. Among those insured by Medicaid, we observed that only half were continuously insured prior to diagnosis, with those discontinuously insured or insured at diagnosis 1.2 to 1.6 times more likely to be diagnosed at a later stage than AYAs who were continuously insured. Those who remained uninsured were approximately 1.2 times more likely to be diagnosed at a later stage than those with private insurance. Sociodemographic factors, including sex, race and ethnicity, and neighborhood SES, were also independently associated with later stage at diagnosis. Overall, our study suggests that lacking continuous health insurance (as demonstrated by variations in Medicaid access) hinders the early detection of cancer.

In our study, obtaining Medicaid at diagnosis, which was consistently associated with a later stage at diagnosis for all cancers considered in this study, or being intermittently enrolled in Medicaid were the most strongly associated with later stage at diagnosis among AYAs. As cancer screening is not recommended for most cancer sites in AYAs, except cervical cancer, identifying cancer early through symptom evaluation and physical examination, the main strategies used in the AYA population (7), requires regular contact with the health care system, something less likely to occur among those without insurance. Further, AYAs commonly present to their primary care provider or emergency department with nonspecific symptoms, often being dismissed as being too young or unlikely to have a cancer diagnosis, which could lead to delays in diagnoses, particularly among the uninsured or underinsured population (16-18). However, AYAs who were continuously insured with Medicaid still had a higher likelihood of later stage at diagnosis for many of the cancers considered in our study, highlighting that other factors (eg, access to paid sick leave, reliable transportation) may influence accessing medical care in this population. Although studies have documented improvements in usual source of care and lower financial barriers to care among the Medicaid (vs uninsured) population, narrow networks or high out-of-pocket costs have been cited as barriers to accessing care for those with Medicaid insurance and should continue to be a focus of future studies (19-21).

Our study expands on prior studies in AYAs that have found that public or no insurance is associated with later stage at diagnosis $(6-8,22)$ by considering Medicaid enrollment prior to diagnosis. Our findings of less pronounced associations with later stage among the uninsured compared to those who obtain Medicaid at diagnosis may reflect Medicaid enrollment at diagnosis being more common among those with advanced stage 


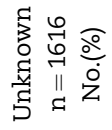

获

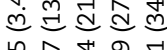

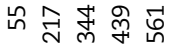

\section{莽}

茫

氙 鵤 है

สํำ

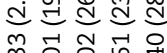

m施舒

สุว

造过

8

产 웅

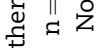

จ สูกิรด

लं

नิ

定

ब궁

过

항스늘

iูi

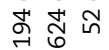

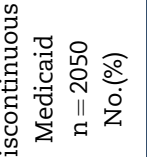

कกิก

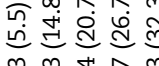

लె

命

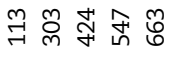

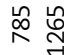

สี

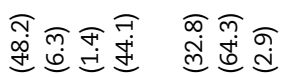

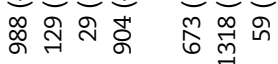

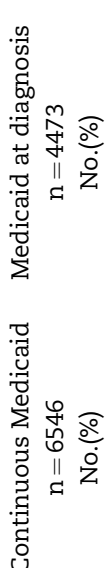

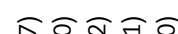

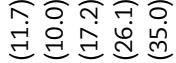

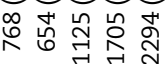

กู่

זิ

잉

본

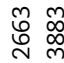

ติ

के व

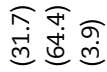

艺芒品

ิํㄱำ

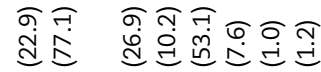

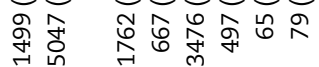

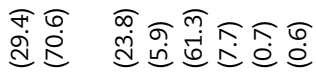

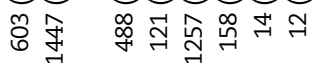

लें

옥

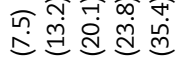

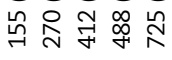

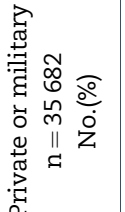

ธดูกิ

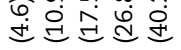

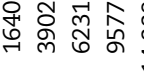

लें

F: 6

式

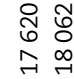

สู่ํํำ

究

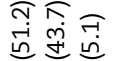

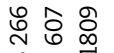

品

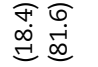

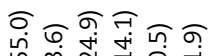

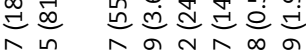

ㄲำ ㄱำ

กิ

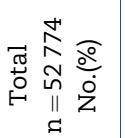

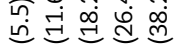

مิ

ธิธ วิธ

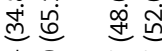

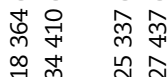

它它君岀

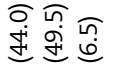

가교

它家 它

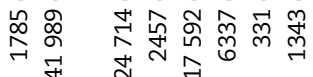

बุ

กิ่

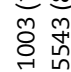

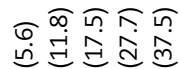

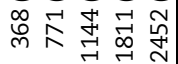

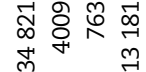

ते क्ष

会 命

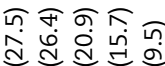

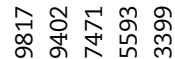

สฺ

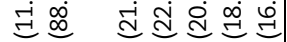

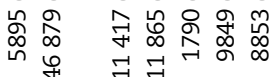

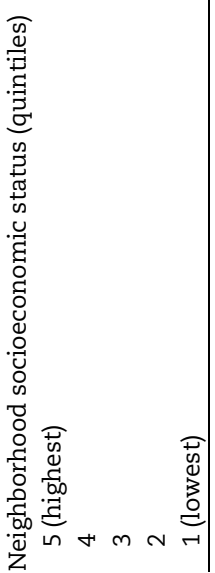




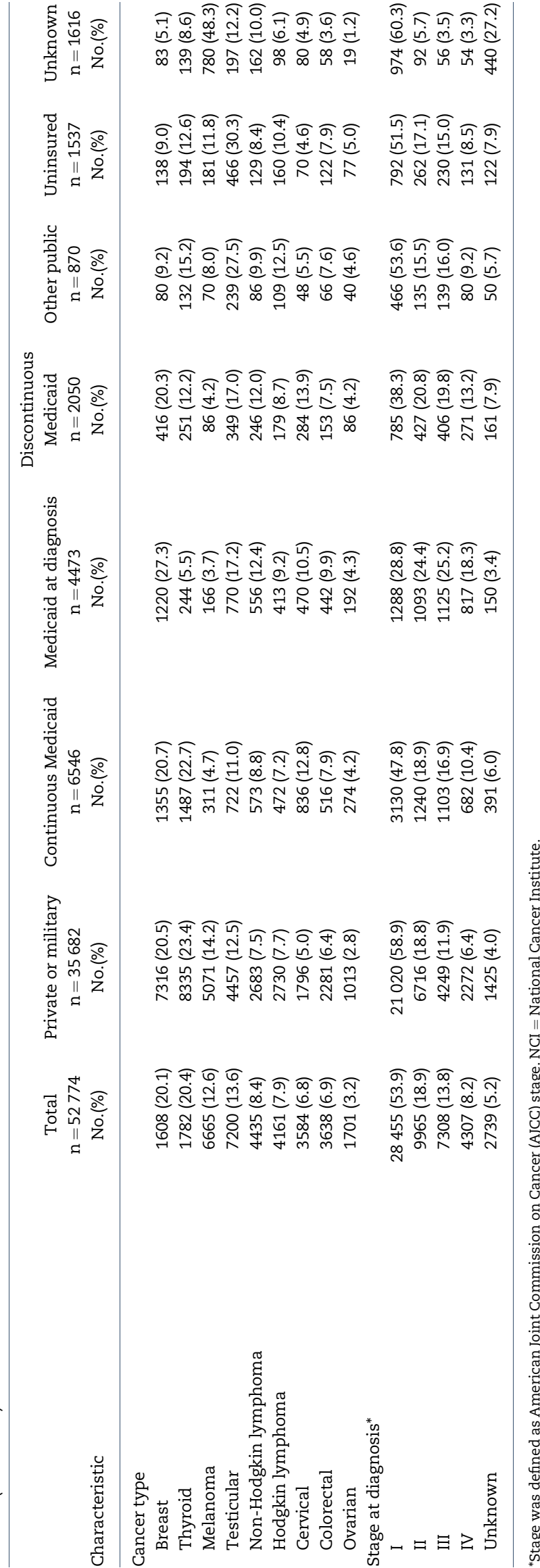

disease, as found previously $(7,8)$. Although ours is the first study to focus on AYAs (to our knowledge), our results are consistent with studies that have considered the timing of Medicaid enrollment in primarily older adult populations (9,23-27). Continuous Medicaid enrollment also was found to provide a survival benefit among older adults with colon, esophageal, ovarian, pancreatic, stomach, or lung cancer, with the observed benefit primarily mediated through earlier stage at diagnosis (9), underscoring the importance of detecting cancers early.

Our findings of a later stage of diagnosis being independently associated with male sex, lower neighborhood SES, and Hispanic or black race and ethnicity are consistent with prior studies $(6,27,28)$. As recently reported $(29)$, we also observed that American Indian/Alaskan Natives were not more likely than non-Hispanic whites to be diagnosed with later stage disease. Although health insurance is an important barrier to receiving timely health care, our findings highlight that other social, cultural, and economic barriers (30-35) impact seeking care that can result in worse cancer survival, as we have observed previously (10).

The percentage of AYAs who remained uninsured and the likelihood of being diagnosed at a later stage was lower in the more recent time period, after the implementation of the dependent coverage expansion of the Patient Protection and Affordable Care Act (ACA), which required insurers to allow children to remain on their parent's insurance plans until the age of 26 years, and the early expansion of Medicaid to low-income adults in California (36). Our results are consistent with prior studies finding increased insurance rates $(37,38)$ and a shift toward early stage disease $(39,40)$ in adults less than 26 years of age. Although not specifically focused on AYAs, recent data after the full implementation of the ACA found a small shift toward the diagnosis of stage I disease in 18- to 64-year-olds from 2011 to 2013 to the fourth quarter of 2014 (41), highlighting the likely positive effects of the ACA provisions on health outcomes and the importance of continuous health insurance. Despite the benefits of the ACA, it also has created challenges (eg, more restrictive access to in-network oncologists, altering prescription drug formularies) that could negatively impact outcomes for vulnerable populations, including AYAs with cancer (21). Although our study did not distinguish between traditional Medicaid enrollees and those gaining coverage under Medicaid expansion, we acknowledge this important area of future research on the ACA's impact.

Our study has some limitations. Although the linkages between Medicaid and the CCR have been used previously (9), the matching process is subject to some uncertainty, potentially resulting in an underestimation of the impact of health insurance on stage at diagnosis. The study also relied on health insurance information from the CCR. Further, our study lacked data on factors, besides insurance, that could impact obtaining timely health care, such as health or cultural beliefs, financial or geographic barriers, or availability of care. Despite these limitations, this study was population-based and included a large diverse population of AYA cancer patients who received their care across all types of institutions, increasing the generalizability of these findings. In addition, our findings were robust across multiple definitions of late stage disease and cancer types. Lastly, our study is one of the first to differentiate continuous Medicaid enrollment from discontinuous enrollment and enrollment at cancer diagnosis among common cancers in young adults, a historically highly uninsured population [with uninsured rates ranging from $28 \%$ to $31 \%$ in 2008 (42)], 
Table 2. Multivariable adjusted odds ratio (OR) and 95\% confidence interval (CI) estimates for characteristics associated with later stage at cancer diagnosis among adolescents and young adults with nine common cancers, 2005-2014, California ( $\mathrm{N}=50$ 035)*

\begin{tabular}{|c|c|c|c|}
\hline Characteristics & No. & $\begin{array}{l}\text { Stage II-IV (vs I) } \\
\text { OR (95\% CI) }\end{array}$ & $\begin{array}{c}\text { Stage III-IV (vs I-II) } \\
\text { OR }(95 \% \text { CI) }\end{array}$ \\
\hline \multicolumn{4}{|l|}{ Age at diagnosis, $\mathrm{y}$} \\
\hline 15-19 & 2784 & Reference & Reference \\
\hline $20-24$ & 5799 & 0.99 (0.88 to 1.13$)$ & 1.05 (0.93 to 1.18$)$ \\
\hline $25-29$ & 9089 & 0.94 (0.83 to 1.06$)$ & 1.02 (0.91 to 1.15$)$ \\
\hline $30-34$ & 13212 & 0.93 (0.83 to 1.05$)$ & 1.01 (0.90 to 1.13$)$ \\
\hline $35-39$ & 19151 & 0.96 (0.85 to 1.08$)$ & 0.99 (0.89 to 1.11$)$ \\
\hline \multicolumn{4}{|l|}{ Sex } \\
\hline Male & 17252 & Reference & Reference \\
\hline Female & 32783 & 0.74 (0.68 to 0.79$)$ & 0.69 (0.65 to 0.74$)$ \\
\hline \multicolumn{4}{|l|}{ Year of diagnosis } \\
\hline Mar 2005-Dec 2009 & 23708 & Reference & Reference \\
\hline Jan 2010-Dec 2014 & 26327 & 0.96 (0.91 to 1.00$)$ & 0.98 (0.93 to 1.02$)$ \\
\hline \multicolumn{4}{|l|}{ Comorbidity } \\
\hline 0 & 33492 & Reference & Reference \\
\hline 1 & 3858 & 1.21 (1.11 to 1.33 ) & 1.17 (1.07 to 1.28$)$ \\
\hline$>1$ & 718 & 1.78 (1.44 to 2.20$)$ & 2.25 (1.87 to 2.70$)$ \\
\hline Missing & 11967 & 0.75 (0.71 to 0.80$)$ & 0.77 (0.72 to 0.82$)$ \\
\hline \multicolumn{4}{|l|}{ Marital status } \\
\hline Married & 22256 & Reference & Reference \\
\hline Not married & 24888 & 1.06 (1.00 to 1.11$)$ & 1.02 (0.97 to 1.08$)$ \\
\hline Unknown & 2891 & 0.74 (0.66 to 0.84$)$ & 0.66 (0.58 to 0.75$)$ \\
\hline \multicolumn{4}{|l|}{ Facility type } \\
\hline NCI-designated & 10475 & Reference & Reference \\
\hline Non NCI-designated & 39560 & 0.62 (0.58 to 0.65$)$ & $0.66(0.63$ to 0.70$)$ \\
\hline \multicolumn{4}{|l|}{ Race and ethnicity } \\
\hline Non-Hispanic white & 23564 & Reference & Reference \\
\hline Non-Hispanic black & 2318 & 1.32 (1.17 to 1.48$)$ & 1.38 (1.24 to 1.53$)$ \\
\hline Hispanic & 16677 & 1.23 (1.16 to 1.30$)$ & 1.12 (1.06 to 1.19$)$ \\
\hline Asian/Pacific Islander & 6079 & 1.03 (0.95 to 1.11$)$ & 0.97 (0.90 to 1.05$)$ \\
\hline American Indian/Alaskan Native & 315 & $0.96(0.72$ to 1.29$)$ & 0.91 (0.68 to 1.23$)$ \\
\hline Other/Unknown & 1082 & 0.39 (0.31 to 0.48$)$ & $0.36(0.27$ to 0.48$)$ \\
\hline \multicolumn{4}{|l|}{ Urban/Rural residence } \\
\hline Urban & 44446 & Reference & Reference \\
\hline Rural & 5589 & 1.00 (0.93 to 1.08$)$ & 0.97 (0.90 to 1.05$)$ \\
\hline \multicolumn{4}{|c|}{ Neighborhood socioeconomic status (quintiles) } \\
\hline 5 (highest) & 8298 & Reference & Reference \\
\hline 4 & 9325 & 1.11 (1.03 to 1.19$)$ & 1.07 (0.99 to 1.15$)$ \\
\hline 3 & 10250 & 1.29 (1.19 to 1.39$)$ & 1.20 (1.11 to 1.29$)$ \\
\hline 2 & 11294 & 1.32 (1.22 to 1.43$)$ & 1.29 (1.20 to 1.40$)$ \\
\hline 1 (lowest) & 10868 & $1.38(1.27$ to 1.51$)$ & 1.40 (1.29 to 1.52$)$ \\
\hline \multicolumn{4}{|l|}{ Health insurance } \\
\hline Private or military & 34257 & Reference & Reference \\
\hline Continuous Medicaid & 6155 & 1.49 (1.37 to 1.61$)$ & 1.41 (1.31 to 1.52$)$ \\
\hline Discontinuous Medicaid & 1889 & 1.93 (1.70 to 2.18 ) & 1.74 (1.56 to 1.95$)$ \\
\hline Medicaid at diagnosis & 4323 & 2.46 (2.26 to 2.69$)$ & 2.16 (2.00 to 2.33$)$ \\
\hline Other public & 820 & 0.98 (0.82 to 1.18$)$ & $1.18(0.99$ to 1.41$)$ \\
\hline Uninsured & 1415 & 1.24 (1.08 to 1.42$)$ & 1.24 (1.08 to 1.43$)$ \\
\hline Unknown & 1176 & 0.63 (0.51 to 0.76$)$ & 0.78 (0.62 to 0.98$)$ \\
\hline
\end{tabular}

*Adjusted for all variables in the table and cancer type. $\mathrm{NCI}=$ National Cancer Institute.

who comprise a large proportion of the Medicaid population in California [41\% of 19- to 44 -year-olds in 2013 (43)].

Overall, our findings suggest that access to continuous medical insurance and care is important for decreasing the likelihood of late stage cancer diagnosis; however, half of AYAs with Medicaid insurance did not have continuous enrollment at the time of our study. Taken together with recent studies demonstrating substantial declines in uninsured rates in Medicaid expansion states corresponding with shifts to earlier stage cancers (41), our findings emphasize the importance of strengthening the Health Insurance Marketplace and Medicaid expansion to increase continuous medical insurance coverage. In addition, strategies that target awareness about Medicaid eligibility, conduct outreach to vulnerable populations, simplify the enrollment process, and educate about affordable plan selection (20) could positively impact access to health care in AYAs. Although lacking continuous health insurance is a substantial barrier to medical care, even with insurance, our 
Table 3. Multivariable adjusted odds ratio (OR) and 95\% confidence interval (CI) estimates for associations between health insurance type and stage at cancer diagnosis among adolescents and young adults, by cancer type, 2005-2014, California ( $\mathrm{N}=50$ 035)*

\begin{tabular}{|c|c|c|c|}
\hline Health insurance type & No. & $\begin{array}{l}\text { Stage II-IV (vs I) } \\
\text { OR (95\% CI) }\end{array}$ & $\begin{array}{c}\text { Stage III-IV (vs I-II) } \\
\text { OR }(95 \% \text { CI })\end{array}$ \\
\hline Breast & 10174 & & \\
\hline Private or military & 7097 & Reference & Reference \\
\hline Continuous Medicaid & 1274 & 1.64 (1.39 to 1.93$)$ & 1.53 (1.33 to 1.76$)$ \\
\hline Discontinuous Medicaid & 373 & 1.97 (1.45 to 2.68 ) & 1.67 (1.33 to 2.10$)$ \\
\hline Medicaid at diagnosis & 1178 & 1.80 (1.52 to 2.14$)$ & 1.77 (1.54 to 2.04$)$ \\
\hline Other public & 73 & 1.45 (0.80 to 2.62$)$ & 2.09 (1.30 to 3.37) \\
\hline Uninsured & 124 & 1.25 (0.81 to 1.93$)$ & 1.44 (0.98 to 2.11$)$ \\
\hline Unknown & 55 & 0.81 (0.45 to 1.48$)$ & 1.62 (0.90 to 2.93$)$ \\
\hline Thyroid & 10648 & & \\
\hline Private or military & 8245 & Reference & Reference \\
\hline Continuous Medicaid & 1472 & 1.32 (0.86 to 2.03 ) & 0.62 (0.28 to 1.40$)$ \\
\hline Discontinuous Medicaid & 247 & $1.66(0.78$ to 3.54$)$ & 2.58 (0.98 to 6.82 ) \\
\hline Medicaid at diagnosis & 241 & $2.42(1.25$ to 4.66$)$ & 2.98 (1.23 to 7.19$)$ \\
\hline Other public & 131 & 1.32 (0.47 to 3.75$)$ & 1.38 (0.29 to 6.68$)$ \\
\hline Uninsured & 192 & 1.33 (0.56 to 3.13$)$ & 1.51 (0.43 to 5.30$)$ \\
\hline Unknown & 120 & 0.87 (0.21 to 3.68$)$ & 0.79 (0.06 to 10.25$)$ \\
\hline Melanoma & 6062 & & \\
\hline Private or military & 4746 & Reference & Reference \\
\hline Continuous Medicaid & 267 & 2.18 (1.63 to 2.91$)$ & 1.86 (1.33 to 2.60$)$ \\
\hline Discontinuous Medicaid & 63 & 3.64 (2.10 to 6.31$)$ & 2.43 (1.35 to 4.37$)$ \\
\hline Medicaid at diagnosis & 158 & 4.18 (2.92 to 5.99$)$ & 4.38 (3.03 to 6.32$)$ \\
\hline Other public & 64 & 2.68 (1.55 to 4.63$)$ & 2.23 (1.22 to 4.08$)$ \\
\hline Uninsured & 161 & 1.39 (0.92 to 2.10$)$ & 1.49 (0.92 to 2.43 ) \\
\hline Unknown & 603 & 0.60 (0.38 to 0.93$)$ & 0.40 (0.19 to 0.83$)$ \\
\hline Testicular & 6803 & & \\
\hline Private or military & 4239 & Reference & Reference \\
\hline Continuous Medicaid & 677 & 1.48 (1.23 to 1.79$)$ & 1.62 (1.30 to 2.03$)$ \\
\hline Discontinuous Medicaid & 322 & 1.96 (1.53 to 2.50$)$ & 2.03 (1.54 to 2.68 ) \\
\hline Medicaid at diagnosis & 742 & 3.31 (2.78 to 3.93 ) & 3.93 (3.26 to 4.75$)$ \\
\hline Other public & 226 & 0.71 (0.51 to 1.00$)$ & $0.80(0.52$ to 1.21$)$ \\
\hline Uninsured & 437 & 0.99 (0.78 to 1.25$)$ & 1.10 (0.83 to 1.46$)$ \\
\hline Unknown & 160 & 0.77 (0.48 to 1.24$)$ & 0.59 (0.30 to 1.16$)$ \\
\hline Non-Hodgkin lymphoma & 4109 & & \\
\hline Private or military & 2531 & Reference & Reference \\
\hline Continuous Medicaid & 516 & 1.27 (1.01 to 1.59$)$ & 1.38 (1.12 to 1.71$)$ \\
\hline Discontinuous Medicaid & 230 & 1.71 (1.24 to 2.35$)$ & 1.52 (1.14 to 2.03$)$ \\
\hline Medicaid at diagnosis & 542 & 1.47 (1.18 to 1.83$)$ & 1.66 (1.35 to 2.02$)$ \\
\hline Other public & 81 & 0.97 (0.60 to 1.55$)$ & 0.92 (0.58 to 1.47$)$ \\
\hline Uninsured & 119 & 1.21 (0.81 to 1.81$)$ & 0.96 (0.65 to 1.42$)$ \\
\hline Unknown & 90 & 1.08 (0.68 to 1.73 ) & 1.35 (0.83 to 2.17 ) \\
\hline Hodgkin lymphoma & 3966 & & \\
\hline Private or military & 2622 & Reference & Reference \\
\hline Continuous Medicaid & 447 & 0.94 (0.66 to 1.34$)$ & 1.17 (0.93 to 1.47$)$ \\
\hline Discontinuous Medicaid & 172 & 1.33 (0.76 to 2.32 ) & 1.66 (1.20 to 2.29 ) \\
\hline Medicaid at diagnosis & 403 & 1.74 (1.15 to 2.65$)$ & 1.67 (1.33 to 2.08$)$ \\
\hline Other public & 109 & 0.77 (0.44 to 1.35$)$ & 1.47 (0.99 to 2.19$)$ \\
\hline Uninsured & 155 & 2.52 (1.21 to 5.24$)$ & 1.35 (0.96 to 1.90$)$ \\
\hline Unknown & 58 & 0.42 (0.22 to 0.80$)$ & 0.92 (0.52 to 1.63$)$ \\
\hline Cervical & 3399 & & \\
\hline Private or military & 1736 & Reference & Reference \\
\hline Continuous Medicaid & 791 & 1.56 (1.27 to 1.91$)$ & 1.42 (1.15 to 1.77$)$ \\
\hline Discontinuous Medicaid & 264 & 1.96 (1.47 to 2.63 ) & 1.71 (1.25 to 2.32$)$ \\
\hline Medicaid at diagnosis & 457 & 2.98 (2.36 to 3.77$)$ & 2.39 (1.88 to 3.05$)$ \\
\hline Other public & 42 & 1.30 (0.66 to 2.56$)$ & 1.26 (0.60 to 2.64 ) \\
\hline Uninsured & 60 & 2.44 (1.42 to 4.17$)$ & 1.45 (0.80 to 2.62 ) \\
\hline Unknown & 49 & 0.87 (0.35 to 2.16$)$ & 1.27 (0.51 to 3.20$)$ \\
\hline Colorectal & 3320 & & \\
\hline Private or military & 2105 & Reference & Reference \\
\hline Continuous Medicaid & 462 & 1.22 (0.90 to 1.64$)$ & 1.05 (0.84 to 1.32 ) \\
\hline Discontinuous Medicaid & 136 & 1.72 (1.00 to 2.97 ) & 1.56 (1.06 to 2.31$)$ \\
\hline
\end{tabular}


Table 3. (continued)

\begin{tabular}{lrrr}
\hline Health insurance type & No. & $\begin{array}{c}\text { Stage II-IV (vs I) } \\
\text { OR (95\% CI) }\end{array}$ & $\begin{array}{c}\text { Stage III-IV (vs I-II) } \\
\text { OR (95\% CI) }\end{array}$ \\
\hline Medicaid at diagnosis & 424 & $3.75(2.37$ to 5.95$)$ & $1.95(1.51$ to 2.52$)$ \\
Other public & 58 & $1.19(0.55$ to 2.59$)$ & $1.12(0.64$ to 1.96$)$ \\
Uninsured & 108 & $1.12(0.65$ to 1.93$)$ & $1.07(0.71$ to 1.62$)$ \\
Unknown & 27 & $0.33(0.14$ to 0.79$)$ & $0.67(0.29$ to 1.53$)$ \\
Ovarian & 1554 & & Reference \\
Private or military & 936 & Reference & $1.49(1.07$ to 2.06$)$ \\
Continuous Medicaid & 249 & $1.45(1.06$ to 1.99$)$ & $1.75(1.08$ to 2.82$)$ \\
Discontinuous Medicaid & 82 & $1.77(1.10$ to 2.85$)$ & $2.51(1.76$ to 3.57$)$ \\
Medicaid at diagnosis & 178 & $2.69(1.88$ to 3.83$)$ & $0.54(0.24$ to 1.23$)$ \\
Other public & 36 & $0.48(0.22$ to 1.06$)$ & $1.65(0.94$ to 2.89$)$ \\
Uninsured & 59 & $1.37(0.79$ to 2.38$)$ & $1.08(0.33$ to 3.54$)$ \\
Unknown & 14 & $0.78(0.24$ to 2.54$)$ & \\
\hline
\end{tabular}

${ }^{*}$ Each cancer site considered separately and adjusted for age group, sex, comorbidity, marital status, facility type, race and ethnicity, urban/rural residence, neighborhood socioeconomic status, and year of diagnosis.

findings highlight that subgroups of AYAs, including males, those residing in lower SES neighborhoods, and blacks and Hispanics, continue to be diagnosed at a later stage. These findings suggest that social, cultural, and economic barriers in addition to health insurance may influence seeking and accessing medical care in this population and should be the focus of future research to reduce sociodemographic disparities in outcomes among AYAs with cancer.

\section{Funding}

This work was supported by the Cancer Research Coordinating Committee (grant number CRR-17-420784). The collection of cancer incidence data used in this study was supported by the California Department of Public Health as part of the statewide cancer reporting program mandated by California Health and Safety Code Section 103885; the National Cancer Institute's Surveillance, Epidemiology and End Results Program under contract HHSN261201000140C awarded to the Cancer Prevention Institute of California, contract HHSN261201000035C awarded to the University of Southern California, and contract HHSN261201000034C awarded to the Public Health Institute; and the Centers for Disease Control and Prevention's National Program of Cancer Registries under agreement U58DP003862-01 awarded to the California Department of Public Health.

\section{Notes}

Affiliations of authors: Center for Oncology Hematology Outcomes Research and Training (COHORT) and Division of Hematology and Oncology, University of California Davis School of Medicine, Sacramento, CA (THMK, TW); Division of Health Policy and Management, University of Minnesota, Minneapolis, MN (HMP); California Cancer Reporting and Epidemiologic Surveillance Program, University of California Davis Health, Institute for Population Health Improvement, Sacramento, CA (YC, FBM, CRM, AP-P, KWK); Department of Emergency Medicine, University of California Davis School of Medicine, Sacramento, CA (KWK); Betty Irene Moore School of Nursing, University of California Davis, Sacramento, CA (KWK).
The funders had no role in the design of the study; the collection, analysis, and interpretation of the data; the writing of the manuscript; or the decision to submit the manuscript for publication. The ideas and opinions expressed herein are those of the author(s), and endorsement by the state of California, Department of Public Health, the National Cancer Institute, and the Centers for Disease Control and Prevention or their contractors and subcontractors is not intended nor should be inferred.

Author contributions: design and conception: THMK, HMP, CRM, AP, KWK, TW; acquisition of data and analysis: FBM, YC; drafting of the manuscript: THMK; final review and approval: THMK, HMP, YC, FBM, CRM, AP, KWK, TW.

Conflicts of interest: KWK is the principal investigator on the Medi-Cal Quality Improvement Program (MCQuIP) funded by the California Department of Health Care Services. The other authors have no disclosures.

A related abstract was presented at the 60th annual meeting of the American Society of Hematology, San Diego, California, December 2018 (Abstract No. 977).

\section{References}

1. Barr RD, Ries LA, Lewis DR, et al. Incidence and incidence trends of the most frequent cancers in adolescent and young adult Americans, including "nonmalignant/noninvasive" tumors. Cancer. 2016;122(7):1000-1008.

2. Bleyer A, Ferrari A, Whelan J, Barr RD. Global assessment of cancer incidence and survival in adolescents and young adults. Pediatr Blood Cancer. 2017;64(9): e26497.

3. Adolescent and Young Adult Oncology Progress Review Group. Closing the Gap: Research and Care Imperatives for Adolescents and Young Adults with Cancer. Bethesda, MD: National Institutes of Health; 2006.

4. Keegan TH, Ries LA, Barr RD, et al. Comparison of cancer survival trends in the United States of adolescents and young adults with those in children and older adults. Cancer. 2016;122(7):1009-1016.

5. Thompson K, Palmer S, Dyson G. Adolescents and young adults: issues in transition from active therapy into follow-up care. Eur J Oncol Nurs. 2009; 13(3):207-212.

6. Aizer AA, Falit B, Mendu ML, et al. Cancer-specific outcomes among young adults without health insurance. J Clin Oncol. 2014;32(19):2025-2030.

7. Robbins AS, Lerro CC, Barr RD. Insurance status and distant-stage disease at diagnosis among adolescent and young adult patients with cancer aged 15 to 39 years: National Cancer Data Base, 2004 through 2010. Cancer. 2014;120(8): $1212-1219$.

8. Rosenberg AR, Kroon L, Chen L, Li CI, Jones B. Insurance status and risk of cancer mortality among adolescents and young adults. Cancer. 2015;121(8): 1279-1286.

9. Dawes AJ, Louie R, Nguyen DK, et al. The impact of continuous Medicaid enrollment on diagnosis, treatment, and survival in six surgical cancers. Health Serv Res. 2014;49(6):1787-1811. 
10. DeRouen MC, Parsons HM, Kent EE, Pollock BH, Keegan T. Sociodemographic disparities in survival for adolescents and young adults with cancer differ by health insurance status. Cancer Causes Control. 2017;28(8):841-851.

11. Barr RD, Holowaty EJ, Birch JM. Classification schemes for tumors diagnosed in adolescents and young adults. Cancer. 2006;106(7):1425-1430.

12. Swerdlow SH, Campo E, Harris NL, Jaffe ES, Pileri SA, Stein H, Thiele J, Vardiman JW. (Eds.): WHO Classification of Tumours of Haematopoietic and Lymphoid Tissues. IARC: Lyon; 2008.

13. Lichtensztajn DY, Giddings BM, Morris CR, Parikh-Patel A, Kizer KW. Comorbidity index in central cancer registries: the value of hospital discharge data. Clin Epidemiol. 2017;9:601-609.

14. Killion JA, Giddings BM, Morris CR, Parikh-Patel A, Kizer KW. Cancer Stage at Diagnosis in California, 2009-2013. Sacramento, CA: California Cancer Reporting and Epidemiologic Surveillance Program, Institute for Population Health Improvement, University of California Davis; 2016. https://www.ucdmc.ucdavis.edu/iphi/Programs/CCR/resources/Cancer\%20Stage\%20at\%20Diagnosis \%20in\%20California\%202009-2013.pdf. Accessed November 2, 2017.

15. Yost K, Perkins C, Cohen R, Morris C, Wright W. Socioeconomic status and breast cancer incidence in California for different race/ethnic groups. Cancer Causes Control. 2001;12(8):703-711.

16. Kent EE, Parry C, Montoya MJ, Sender LS, Morris RA, Anton-Culver H. "You're too young for this": adolescent and young adults' perspectives on cancer survivorship. J Psychosoc Oncol. 2012;30(2):260-279.

17. Xu Y, Stavrides-Eid M, Baig A, et al. Quantifying treatment delays in adolescents and young adults with cancer at McGill University. Curr Oncol. 2015 22(6):470-477.

18. Martin S, Ulrich C, Munsell M, Taylor S, Lange G, Bleyer A. Delays in cancer diagnosis in underinsured young adults and older adolescents. Oncologist. 2007; 12(7):816-824.

19. Kirby JB, Vistnes JP. Access to care improved for people who gained Medicaid or marketplace coverage in 2014. Health Aff (Millwood). 2016;35(10):1830-1834.

20. Kominski GF, Nonzee NJ, Sorensen A. The Affordable Care Act's impacts on access to insurance and health care for low-income populations. Annu Rev Public Health. 2017;38:489-505.

21. Hsu P, Graves JA. The Affordable Care Act and adolescents and young adults with cancer: much progress, but challenges remain. JAMA Oncol. 2018;4(8):1043-1044.

22. Smith EC, Ziogas A, Anton-Culver H. Association between insurance and socioeconomic status and risk of advanced stage Hodgkin lymphoma in adolescents and young adults. Cancer. 2012;118(24):6179-6187.

23. O'Malley CD, Shema SJ, Clarke LS, Clarke CA, Perkins CI. Medicaid status and stage at diagnosis of cervical cancer. Am J Public Health. 2006;96(12): 2179-2185.

24. Perkins CI, Wright WE, Allen M, Samuels SJ, Romano PS. Breast cancer stage at diagnosis in relation to duration of Medicaid enrollment. Med Care. 2001; 39(11):1224-1233.

25. Bradley CJ, Given CW, Roberts C. Late stage cancers in a Medicaid-insured population. Med Care. 2003;41(6):722-728.

26. Koroukian SM. Assessing the effectiveness of Medicaid in breast and cervical cancer prevention. J Public Health Manag Pract. 2003;9(4):306-314.

27. Pollitt RA, Clarke CA, Shema SJ, Swetter SM. California Medicaid enrollment and melanoma stage at diagnosis: a population-based study. Am J Prev Med. 2008;35(1):7-13
28. Halpern MT, Ward EM, Pavluck AL, Schrag NM, Bian J, Chen AY. Association of insurance status and ethnicity with cancer stage at diagnosis for 12 cancer sites: a retrospective analysis. Lancet Oncol. 2008;9(3):222-231.

29. Adams SV, Burnett-Hartman AN, Karnopp A, et al. Cancer stage in American Indians and Alaska natives enrolled in Medicaid. Am J Prev Med. 2016;51(3): 368-372.

30. Kullgren JT, McLaughlin CG, Mitra N, Armstrong K. Nonfinancial barriers and access to care for U.S. adults. Health Serv Res. 2012;47(1, pt 2):462-485.

31. Guendelman S, Schauffler H, Samuels S. Differential access and utilization of health services by immigrant and native-born children in working poor families in California. J Health Care Poor Underserved. 2002;13(1):12-23.

32. Jones CE, Maben J, Jack RH, et al. A systematic review of barriers to early presentation and diagnosis with breast cancer among black women. BMJ Open. 2014;4(2):e004076.

33. Nair M, Baltag V, Bose $\mathrm{K}$, Boschi-Pinto C, Lambrechts $\mathrm{T}$, Mathai M. Improving the quality of health care services for adolescents, globally: a standardsdriven approach. J Adolesc Health. 2015;57(3):288-298.

34. Akinlotan M, Bolin JN, Helduser J, Ojinnaka C, Lichorad A, McClellan D. Cervical cancer screening barriers and risk factor knowledge among uninsured women. J Community Health. 2017;42(4):770-778.

35. Zhou J, Enewold L, Peoples GE, et al. Trends in cancer screening among Hispanic and white non-Hispanic women, 2000-2005. J Womens Health (Larchmt). 2010;19(12):2167-2174

36. Golberstein E, Gonzales G, Sommers BD. California's early ACA expansion increased coverage and reduced out-of-pocket spending for the state's lowincome population. Health Aff (Millwood). 2015;34(10):1688-1694.

37. Parsons HM, Schmidt S, Tenner LL, Bang H, Keegan TH. Early impact of the Patient Protection and Affordable Care Act on insurance among young adults with cancer: analysis of the dependent insurance provision. Cancer. 2016;122:1766-1773.

38. Alvarez EM, Keegan TH, Johnston EE, et al. The Patient Protection and Affordable Care Act dependent coverage expansion: disparities in impact among young adult oncology patients. Cancer. 2018;124(1):110-117.

39. Han X, Zang Xiong K, Kramer MR, Jemal A. The Affordable Care Act and cancer stage at diagnosis among young adults. J Natl Cancer Inst. 2016;108(9): djw058.[CrossRef]

40. Robbins AS, Han X, Ward EM, Simard EP, Zheng Z, Jemal A. Association between the Affordable Care Act dependent coverage expansion and cervical cancer stage and treatment in young women. JAMA. 2015;314(20):2189-2191.

41. Jemal A, Lin CC, Davidoff AJ, Han X. Changes in insurance coverage and stage at diagnosis among nonelderly patients with cancer after the Affordable Care Act. J Clin Oncol. 2017;35(35):3906-3915.

42. Rodean J. Health insurance coverage of young adults aged 19 to 25: 2008, 2009, and 2011. American Community Survey Briefs. 2012. https://www2.census.gov/library/publications/2012/acs/acsbr11-11.pdf.. Accessed March 20, 2013

43. Medicaid enrollment by age. Henry J Kaiser Family Foundation website. https:// www.kff.org/medicaid/state-indicator/medicaid-enrollment-by-age/? currentTimeframe=0\&sortModel=\%7B\%22colId\%22:\%22Location\%22,\%22sort \%22:\%22asc\%22\%7D. Accessed November 5, 2018. 